\title{
Designing diagrams for Wikipedia
}

Keywords: diagrams, Wikipedia, collaborative project, design education, Information visualization, image contribution

Despite the high usage of diagrams and images in Wikipedia, as well as across all Wikimedia projects, few studies have been conducted on the role of visual contents within the online encyclopedia and on the collaborative creation of diagrams. This article describes research that brought a group of designers into Wikipedia, with the goal of exploring how information designers act on this platform and how other users react to their involvement. Inspired by the WikiEdu Program, we engaged postgraduate design students in the creation of diagrams for Wikipedia and then followed the reactions of both users and designers to their work. The results of the experiment have been evaluated using built-in Wikipedia functions (e.g., page history and discussion pages) and through an anonymous survey among the students involved. This experience brought to light the different consideration granted to images and diagrams with respect to texts on the online encyclopedia. It also allowed a reflection on the role of designers in the knowledge production process, as well as on the meaning of producing "open" contents, which are meant to be improved by other people.

\section{Introduction}

Today, Wikipedia represents one of the main access points to information: it is the 6th most visited site in the world (Alexa Internet Inc. 2016), and at the moment of writing it counts 294 language editions ("List of Wikipedias" 2016). Its value goes beyond the publicly displayed information through its interface. The whole process of the creation and refinement of information, the discussions about its policies, and the social relationships behind the project have been stored and made publicly available. The result is one of the most intriguing datasets on the collaborative creation of knowledge and meaning on earth. Moreover, all this information is free from copyright and without technical limits for its reuse.

Quite a solid body of research is taking shape around Wikipedia as a significant data source, thus suggesting the need to apply analysis and visualization techniques to extract and make sense of massive amounts of information (Bao et al. 2012; Massa \& Scrinzi 2013; Wattenberg, Viégas \& Hollenbach 2007). However, very little research has been conducted on the role of images and diagrams within Wikipedia articles and the collaborative process of their creation (Viegas 2007). Nevertheless images and diagrams are a relevant part of the Wikipedia ecosystem: Creative Commons, Wikipedia's side project focused on media, counts more than one billion media items ("State 
of the Commons" 2015) used across all Wikipedia-related projects. Images and diagrams are indeed a powerful device for information (Ricci 2010) and users rely on them just as much as on text, if not more.

By recognizing this dearth of scientific production, the present research brought a group of designers into Wikipedia to analyze how information designers act on Wikipedia and how Wikipedia users react to their involvement.

To reach the needed critical mass for a similar experiment, we involved students on their second year of a master's degree in Communication Design, thus young professionals. Inspired by the WikiEdu Program, we asked our students to design a diagram for a Wikipedia article. The WikiEdu Program is simple and effective: as an assignment, students need to improve a Wikipedia page related to the course's topic ("WikiEdu Website," n.d.). By doing so the students develop writing skills and critical thinking, and at the same time they produce something valuable for the whole community. We saw a great potential in bringing this kind of assignment into graphic design education, specifically in information design. This assignment allowed us to investigate the relationship between Wikipedia and diagrams from two different angles:

- An analysis of how information designers act on Wikipedia, and how Wikipedia users react to designers' involvement. As we will explain later, the assignment's goal was to produce a diagram for a specific Wikipedia page, and for the final evaluation students had to engage with other users to include the diagram in the article.

- The definition of a new format for design education. We transposed the WikiEdu guidelines from the creation of texts to the creation of diagrams, seeing it as an enriching experience both for students and for the Wikipedia community. The experience was a test to understand whether a format that proved to be valuable for texts could also work in the field of information design.

In this article, we will focus mainly on the first angle.

\section{Experiment setup}

The formulation of the assignment was designed to follow the basic rule of Wikipedia: write about something you know. Thus each student was encouraged to select a well-known topic so as to evaluate if there was a lack of diagrams in the related article. Since "diagram" can have a vast range of meanings (Ricci, Ciuccarelli \& Valsecchi 2008), we suggested focusing on a topic belonging to these four categories:

- Processes: the making of something, phases, materials, and techniques;

- Technological descriptions: how something works, its components and the relationships among them;

- Historical events: temporal evolution of an event, the main actors, their movements

- Quantitative phenomena: create a visual representation of a dataset, highlighting relevant correlations.

The diagram had to be designed for a specific existing article. This made it simpler for students to identify which part of the topic should be visualized and what kind of knowledge could they add to it. Students were free to create a new diagram or to improve an existing one within the article. Since on Wikipedia there are some meta-pages containing lists of requested materials, we pointed the students to the list of requested diagrams ${ }^{1}$ so that they could understand what types of diagrams are often requested by users. Finally, no technical constraints were given: students were free to choose any visual language and technique. 
Students had seven weeks to create the diagram, upload it, and advocate for its inclusion. Preparing this assignment, we took advantage of the knowledge gathered through other projects related to Wikipedia (Borra et al. 2015; Mauri 2011). These experiences allowed us to better understand how the encyclopedia works, particularly its social dynamics and its policies, formalizing them in the following four guidelines we gave to the students.

\subsection{Understand the project}

Wikipedia is just the most visible part of a much bigger ecosystem of projects, operated by Wikimedia Foundation. ${ }^{2}$ Each project focuses on a specific type of knowledge: Wikiquote for quotation, Wikisource for historical books, Wikinews for current topic, Wikipedia for the encyclopedic knowledge, and so on.

Wikimedia Commons is the project focused on media: images, videos, and audio files are collected there. Images can be then linked on any Wikipedia page, in any language. Before uploading images, our students had to become familiar with the projects and understand their dynamics.

\subsection{Follow the standards}

There are no clear standards on Wikipedia about images. The only requirement is to adopt a free license, such as a Creative Commons license. We asked students to identify whether best practices exist and are already shared among Wikipedia users. It turned out that while there are several guidelines for articles (e.g., "Wikipedia: Policies and guidelines" 2016), little information is available for designing graphics. Only for geographic maps are there community-defined conventions. Even if this could be seen as a drawback, as our students had no clear instruction on how to create diagrams, it pushed them to experiment with information visualization.

\subsection{Follow the ethics}

The third guideline is the most important. Despite the lack of strict standards, we asked the students to find technical solutions to preserve Wikipedia's ethics. Wikipedia presents itself as the "encyclopedia anyone can edit." This also means that anyone can improve its contents and that contributors must distance themselves from the idea of being the recognized authors. As it will be described later, this is a very sensitive topic for graphic designers. In technical terms, this means, for example, that vector formats should be favored over raster images, that text paths should be preferred over traced texts, and that the file layering should be understandable by other people.

\subsection{Engage with the community}

Finally, we asked the student to actively engage with the community: after the diagram has been created, they must upload it and try to push the insertion of the graphic in the related Wikipedia article, engaging with the other users on the talk pages.

\section{Evaluation}

To evaluate the reaction of other users, we repurposed the technical and social mechanisms underlying the collaborative encyclopedia: analysis of discussions, analysis of page history, analysis of diagram reuse, analysis of diagram modifications, and remixes. Below, we briefly introduce the Wikipedia technical features we used for the evaluation. 


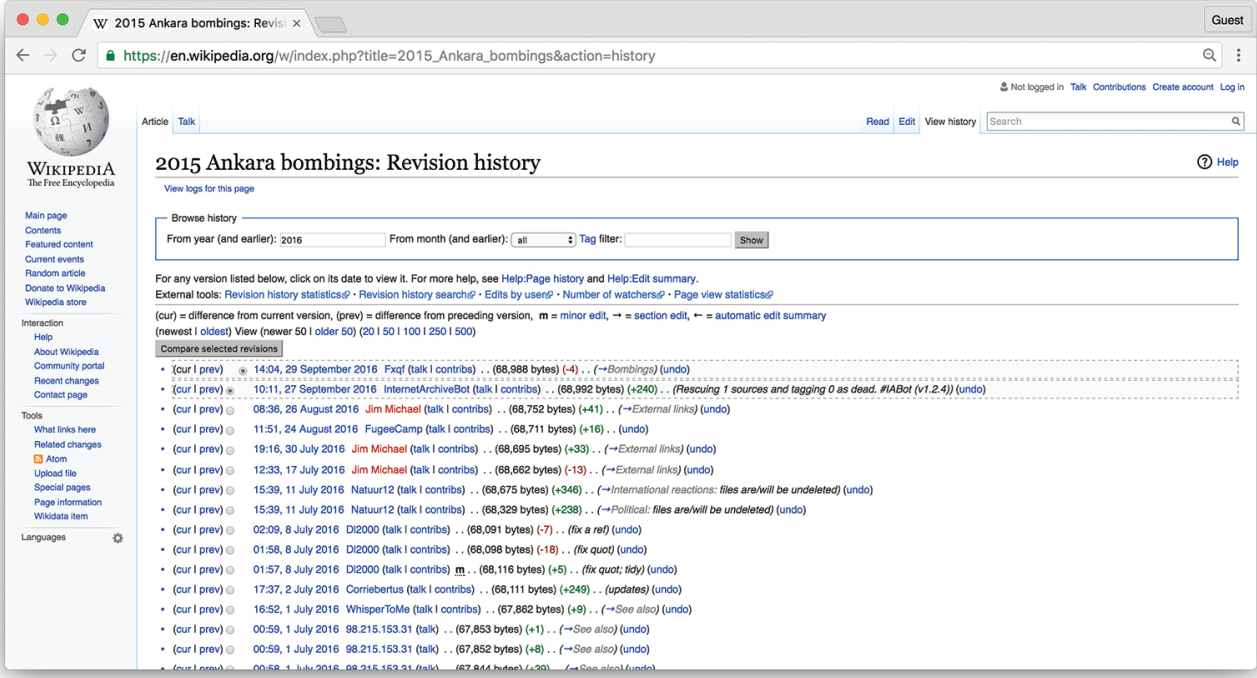

Figure 1. A screenshot of the page history for the "2015 Ankara Bombings" article. It is possible to see the list of edits: for each one it is possible to see the timestamp, the user, and the description.

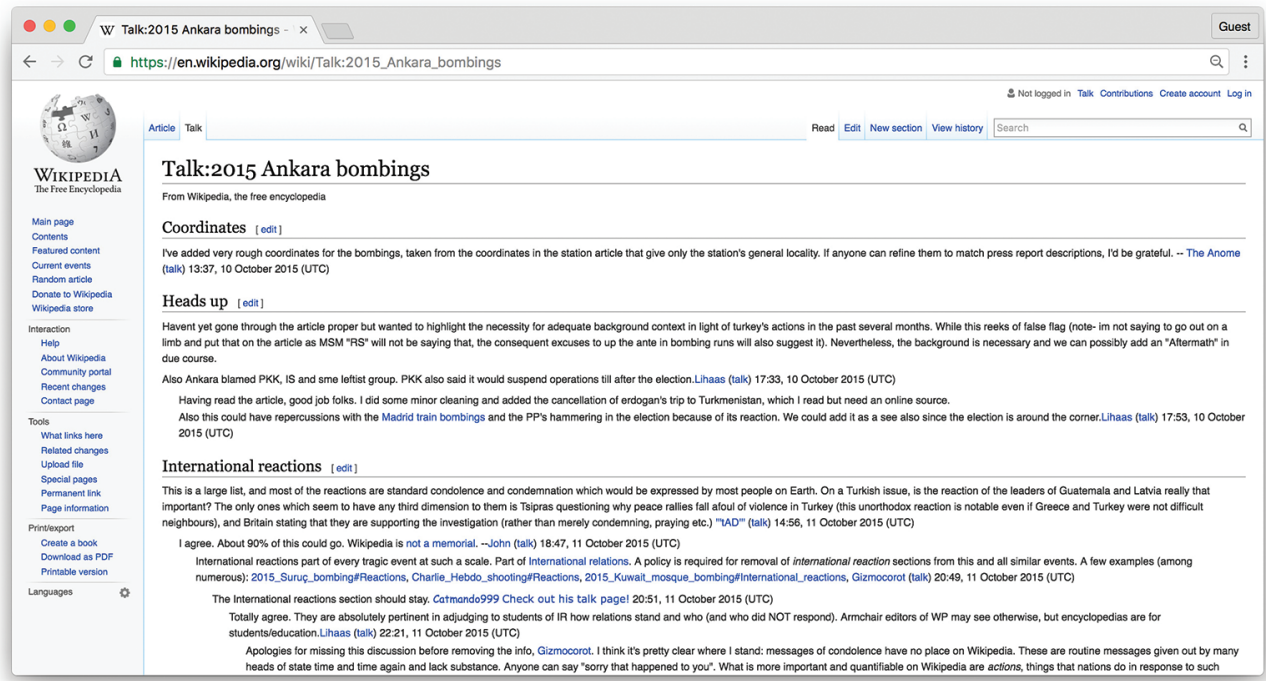

Figure 2. A screenshot of the discussion page for the "2015 Ankara Bombings" article. Each title defines a new discussion, each comment has a different indentation according to the reply chain. 


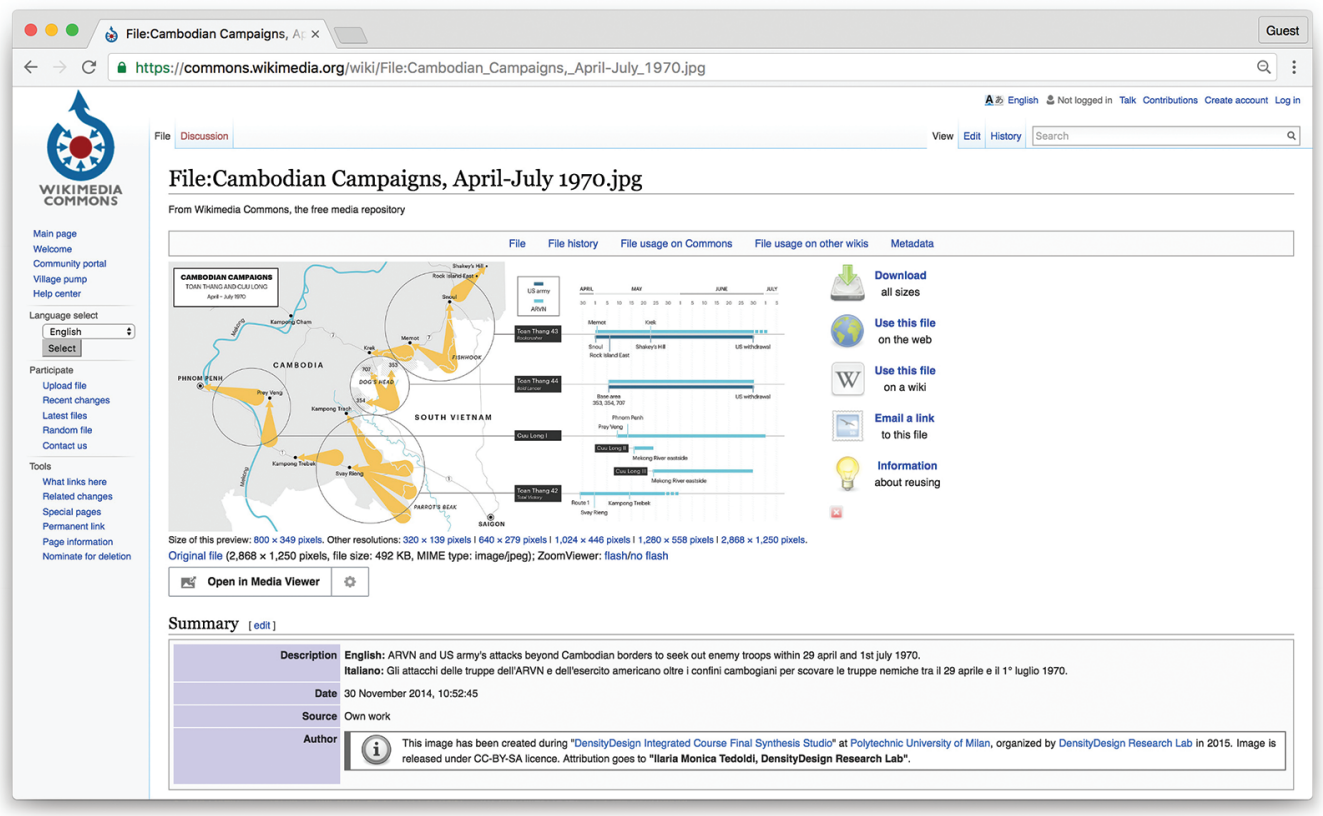

Figure 3. A screenshot of the image page on Wikimedia Commons.

\subsection{Page history}

All the revisions of each Wikipedia article are stored online, and they are publicly available. It is possible to see the revision history in Figure 1. This is originally intended as a versioning tool for editors, such that one can identify the latest edits, who made them and when, compare the latest version with the previous one, and "roll it back" if needed, to restore an old version of the page. Each time a user saves a new version of the page, the system asks for a short description of the edits. In addition, when new images are added or removed, the edit is traced. The revision history of an article is useful to determine whether images were deleted and for what reason, how long they lasted, and the pace of editing for a specific page.

\subsection{Discussion pages}

Each Wikipedia entry is composed of the article page and the discussion page. The "talk" tab opens the discussion page-as in Figure 2-where users coordinate the evolution of an article, and, if needed, solve controversies to avoid edit wars. ${ }^{3}$ The discussion pages are organized in a tree structure to show which comments respond to whom. Commonly, new topics are defined by a new title. When commenting, users leave their signature, meaning a link to their user page and the timestamp of the comment. The edit history of discussion pages, as for the articles, is stored and browsable. Discussion pages were useful to understand why some diagrams were rejected or moved to other pages. We encouraged the students to actively engage 
in discussions, explain the reason for their designs, and accept suggestions and critiques.

\subsection{Image pages}

Images are stored in the Wikimedia Commons website, the "sister project" focused on media (pictures, videos, sounds). When a new image is uploaded, a wiki-page is created, as in Figure 3. The new page shares all the basic characteristics of articles: history and talk pages. There are some additional functions to image pages, such as the revision history of the picture and the link to all Wikipedia articles using it. These pages are useful to trace the usage of images, their placement in other pages, including linguistic versions, whether derivate works have been created, and whether other users have modified and improved them.

\subsection{Poll among students}

Finally, to collect feedback from the information design community, we asked our students, as a representative sample, to fill up a form anonymously. In the poll, we asked for feedback on their experience regarding whether they found the contribution to Wikipedia interesting, the main difficulties, and their relationship with the project beyond this exercise.

\section{Results}

The students produced and uploaded 73 different diagrams on Wikimedia Commons. ${ }^{4}$ Students covered a huge variety of topics, creating both still images and animations. The diagrams are mainly about processes (such as the production of Jack Daniel, olive oil extraction, etc.), technical descriptions (how an astrolabe works, why we see mirages), and historical events (the Battle of Thermopylae, or the Cambodian Campaign of
1970). Few students worked on "classical" data visualizations (e.g., the evolution of Haitian economy, the results of Olympics Games). Students created both static images (56) and animations (17). Static images are mainly in vector format: only a third of the diagrams are PNG or JPG.

As in Figure 4, the community accepted 45 diagrams, while 13 were rejected. In 15 cases, students were not able to add the image to a Wikipedia article.

We found a correlation between the grades we assigned to the diagrams and their performance on Wikipedia: the lowest graded diagrams were also not accepted in their target article.

\subsection{Debate}

Very few diagrams generated an actual debate in Wikipedia talk pages. All 13 rejected diagrams were at least commented on, while only 8 of the accepted ones created some debate. In the next session we explore in detail the discussion topics (See section 6 "Analysis of Results").
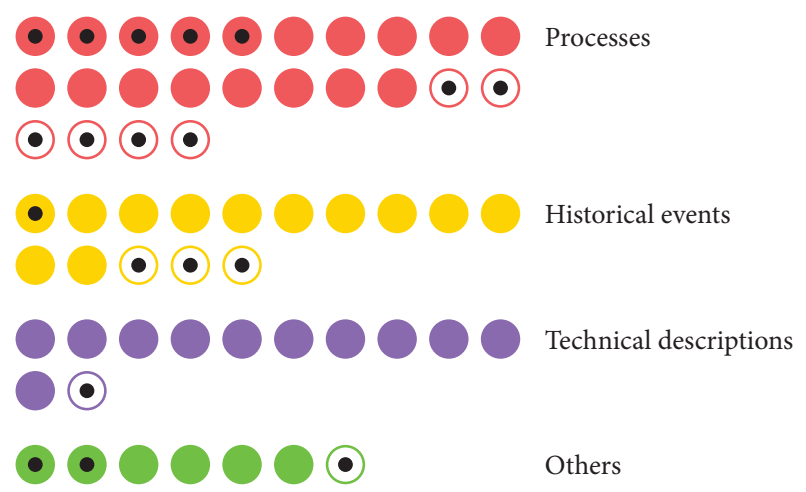

Legend: $\bigcirc$ accepted diagram, $\bigcirc$ rejected diagram, $\bullet$ commented

Figure 4. Accepted, rejected, and discussed diagrams per topic. 


\subsection{Re-use of uploaded diagrams}

Fifteen images were reused on other pages. In most of the cases, diagrams were added to another linguistic version of the same article. Apparently, only one diagram was fully translated into another language. We are saying "apparently" because there are no automatic ways to follow potential derivative works on Wikimedia Commons. When users download and modify an image and then re-upload it, they should put a link to the original work, but this is not automatic.

\section{Analysis of Results}

\subsection{Type of discussion}

Most of the diagrams were accepted without any kind of comment. At the same time, we can identify the main concerns of other users from the few discussions that arose and the main reason for an image's rejection. Four main types of discussions emerged.

5.1.1 Misleading/confusing. Some diagrams were rejected based on the argument that they were not clear or even misleading. Three images received this kind of comment, and as teachers we agreed: The graphics were indeed not visually efficient or the information source was not clear.

5.1.2 Does not add information. If the diagram is simply a visual translation of the text, it will probably be discarded. On Wikipedia, apparently, the text has priority over images. This makes sense in terms of how easy it is to translate, transform, and repurpose text rather than images, especially in the process of adaptation for different supports (mobile, desktop, etc.) or for visually impaired people. Many images have not been accepted because they were mere visual translations of the information contained in the text. Figure 5, for example, represents how the production process of chocolate works. It was refused because, according to users, the space for this image would be better used for a textual description:

I don't think this diagram adds enough to make it worth the page space it will take up. (Wikipedia user, commenting on the "Chocolate Production" diagram)

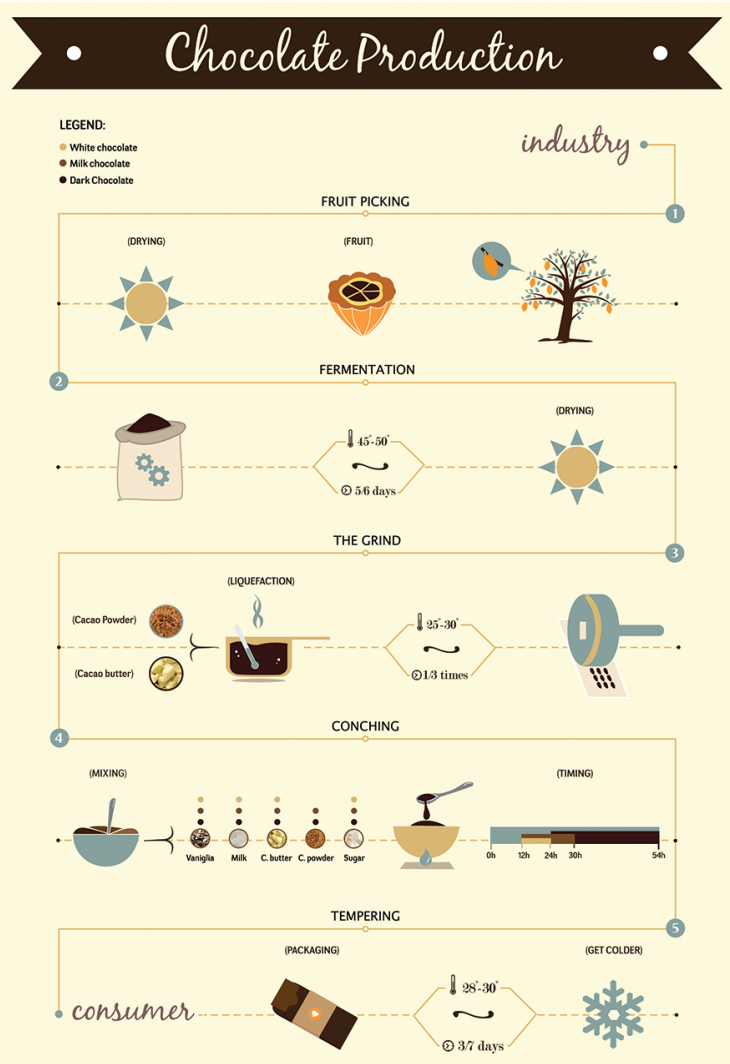

Figure 5. Diagram representing the chocolate production process. 
A second example is Figure 6, refused because it is too illustrative and seen as a decoration rather than real information:

I'm not seeing an advantage to the reader in replacing the current interactive graph with the new one. It looks prettier, but is less helpful. I think if there were a way of introducing graphics and color into the present graph that would be great! (Wikipedia user, commenting on the "Brewing Process" diagram)

5.1.3 Not suited for Wikipedia UI. In the actual interface of Wikipedia, images are usually rendered as 220 pixel wide thumbnails. This means that diagrams meant to be read

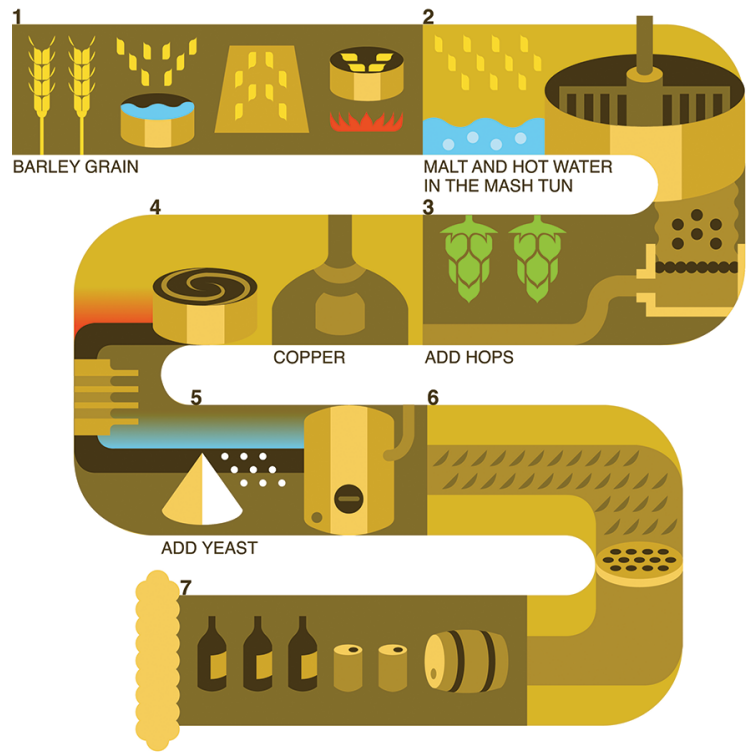

1-MALTING 2-MASHING 3-LAUTERING 4-BOILING 5-FILTERING 6-FERMENTING 7-PACKAGING

Figure 6. Diagram representing the beer brewing process. at full size risk becoming completely illegible. For example, for the diagram in Figure 7 , a user commented:

The main reason for not adding this to the article is that it does not work well as a thumbnail. As the image on the right shows, it doesn't work well as a thumbnail on a web page. Even the clickable version is not easy to read, as the text is too small. It would work best printed on an A4 sheet of paper. (Wikipedia user, commenting on the "Google Acquisitions" diagram)

This kind of critique raises a general question: Should diagrams be designed for the current Wikipedia UI, or can they go beyond it?

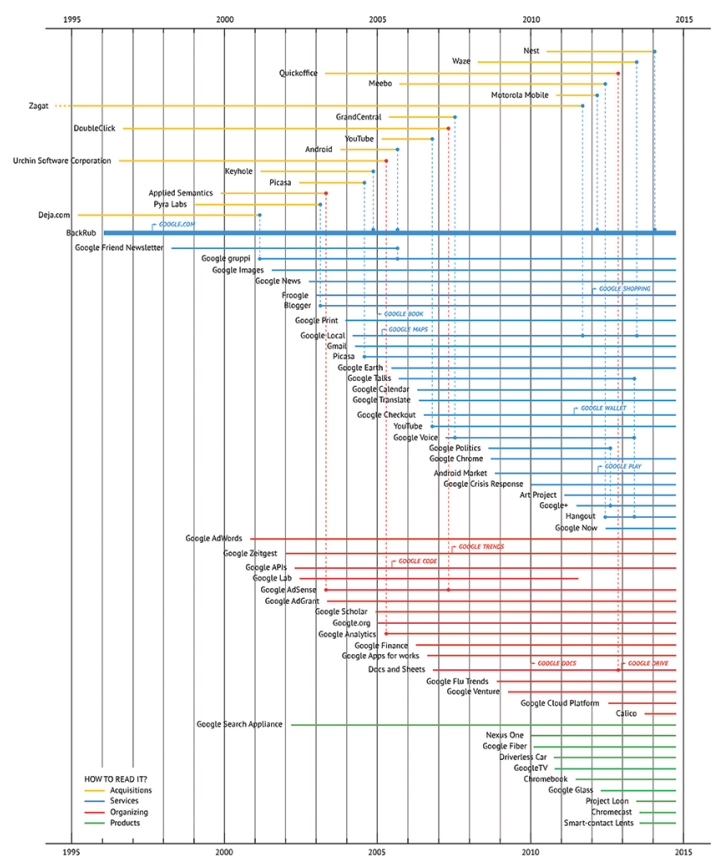

Figure 7. Timeline of the acquisition made by Google, colored by typology. 
5.1.4 Original research. In two cases, the discussion focused on Wikipedia's "no original research" policy (also called WP: NOR):

Wikipedia does not publish original thought: all material in Wikipedia must be attributable to a reliable, published source. Articles may not contain any new analysis or synthesis of published material that serves to advance a position not clearly advanced by the sources. ("Wikipedia: No original research" 2016)

Since many people edit Wikipedia, and it is not possible to verify their competences, every user is required to cite a proper source for the claims added to the page. A person cannot insert his own analysis of a topic: he should first publish it in a journal, conference or known newspaper and then add it to Wikipedia. This rule applies also to images, but users are allowed to create original work:
Original images created by a Wikipedian are not considered original research, so long as they do not illustrate or introduce unpublished ideas or arguments, the core reason behind the NOR (No Original Research) policy. ("Wikipedia: Policies and guidelines" 2016)

The diagram in Figure 8 was rejected for this reason. The diagram represents Odysseus' journey on a map, showing with the thickness of the line the number of men accompanying him. The user rejecting it stated:

[...] I've removed it. No one knows whether the places mentioned in the Odyssey were meant to correspond to real geographical locations, so any map like this would be highly speculative at best. In any case, and more importantly, such a map, not coming from a reliable source would constitute original research which is not allowed on Wikipedia. (Wikipedia user, commenting on the "Possible causes of Colony Collapse Disorder" diagram)

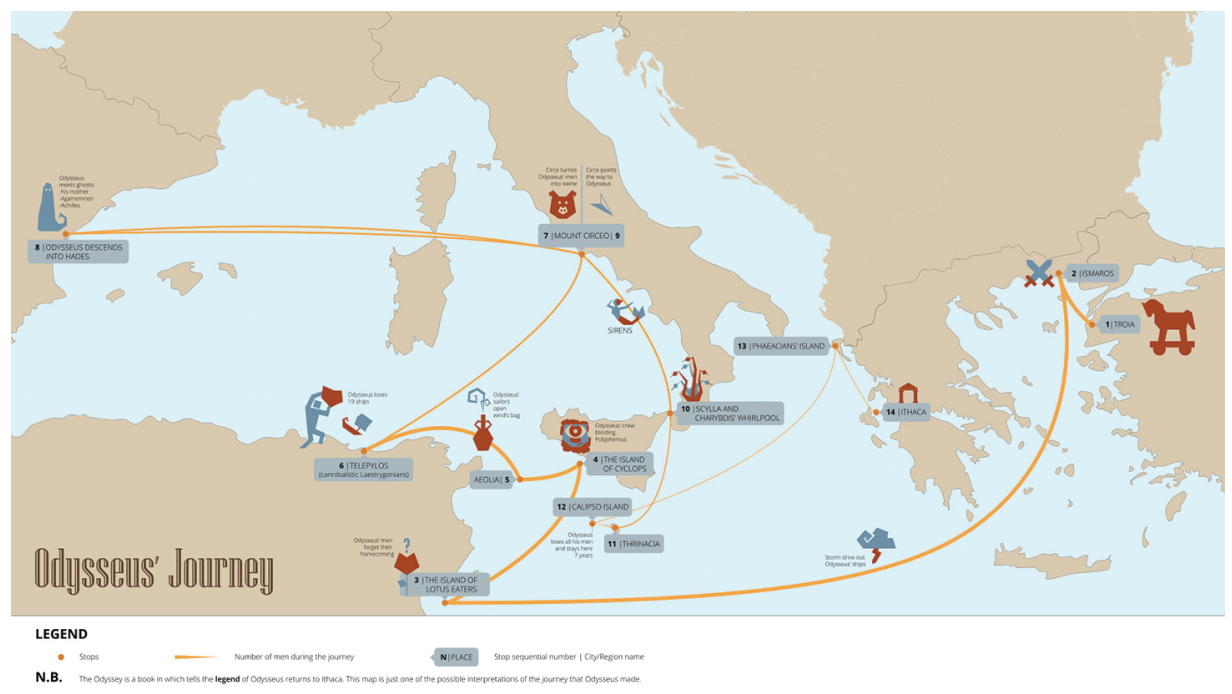

Figure 8. A possible interpretation of Odysseus' journey. 
The diagram "Possible causes of Colony Collapse Disorder” (Figure 9) generated a more complex discussion. In this case, the diagram was a visual translation of the possible causes listed on the page. This became an interesting discussion about how these policies should apply to images:

That the image is based on the article is actually a bit of the problem. What you've basically done is a novel synthesis not only of the subject matter, but we also should not be using Wikipedia as a source (WP: CIRC). If we were going to have such an image, it would need to have been published somewhere, as [previous user] alluded to, so we could verify it came from a source with expertise to review this topic. The effort is definitely appreciated though, as I'm sure some time went into making the image. (Wikipedia user, commenting on the "Possible causes of Colony Collapse Disorder" diagram)
A second user disagreed with him:

I think it should go into the article, until we have a better replacement. My understanding is that the WP: IMPERFECT policy allows imperfect things in the articles. (Wikipedia user, commenting on the "Possible causes of Colony Collapse Disorder” diagram)

In the end, the diagram was not added to the article. This, however, was not due to the "no original research" policy, but rather because images are more difficult to edit than text:

That policy you mention applies much more to articles that we can easily improve over time. That spirit relates more to starting with something sub-standard, but acceptable that can be improved over time. When a flaw is pointed out from the beginning though, we don't need to automatically accept that. The other problem is that

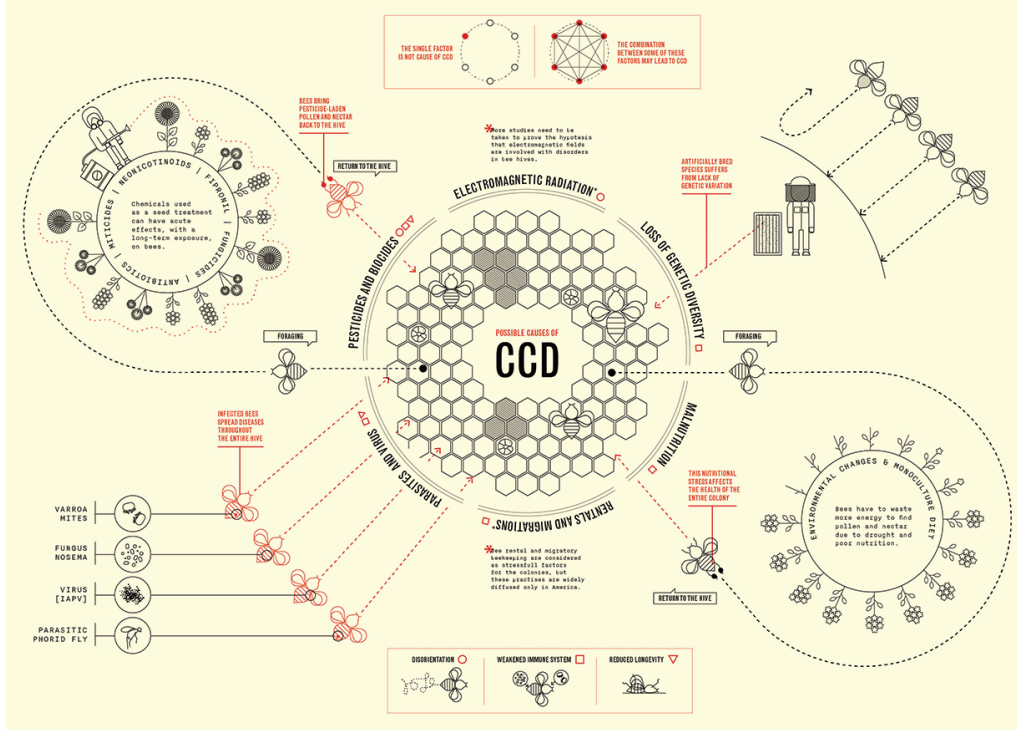

Figure 9. Visual synthesis of "Possible causes of Colony Collapse Disorder." 
images cannot be easily edited like text in an article. You need access to GIS software for this. (Wikipedia user, commenting on the "Possible causes of Colony Collapse Disorder" diagram)

\subsection{Students' feedback}

At the end of the course, we asked students to participate in an anonymous survey to understand whether they found the exercise interesting, the main difficulties, and the unexpected results. Out of 74 students, 32 agreed to participate in the survey. Of these, $84 \%$ said that the creation of a diagram specifically for Wikipedia and not for other uses was an enriching experience for them. The main reasons given for that were that they felt they were contributing to someone else's enrichment, and that they felt responsible for the quality of their work:

You have to design something for everybody. It's an extra responsibility for you. (Anonymous student \#18)

Other students said that it was interesting to understand what is beyond Wikipedia (its dynamics and rules) and that they had the feeling of "giving back" something to a project they use daily, as stated by this student:

At first I didn't understand the utility of this exercise. When it "forced" me to enter into the Wikipedia community and to understand the underlying dynamics, then I found it extremely interesting. (Anonymous student \#8)

Finally, few students said that having a diagram accepted on Wikipedia is valuable for their portfolio visibility.

When discussing the difficulties, $31 \%$ found difficulties in contributing to Wikipedia. Most said that the main difficulty is the use of the SVG format, as MediaWiki (the software running Wikipedia) can render only a few graphic filters and effects. In particular, a strong limitation is the small number of available fonts: even if one can use any font in an SVG image, MediaWiki is able to render only a few of them. Sometimes, even for available fonts, the render is not perfect.

Other difficulties related the choice of a topic. Some students only realized the complexity of their choice after they had started visualizing it:

The chosen topic, in hindsight, was too complex and historically not clear; therefore, the project realization was complex, and in the end, it was not accepted. (Anonymous student \#5)

When asked if they found the exercise useful for their design education, most students said that they found it very useful (see Figure 10). Finally, when asked whether there was something unexpected (positively or negatively) in this experience, most of the students said that working on Wikipedia had been enriching beyond what

Have you found useful the exercise?

$(1=$ useless, 5 = very useful)

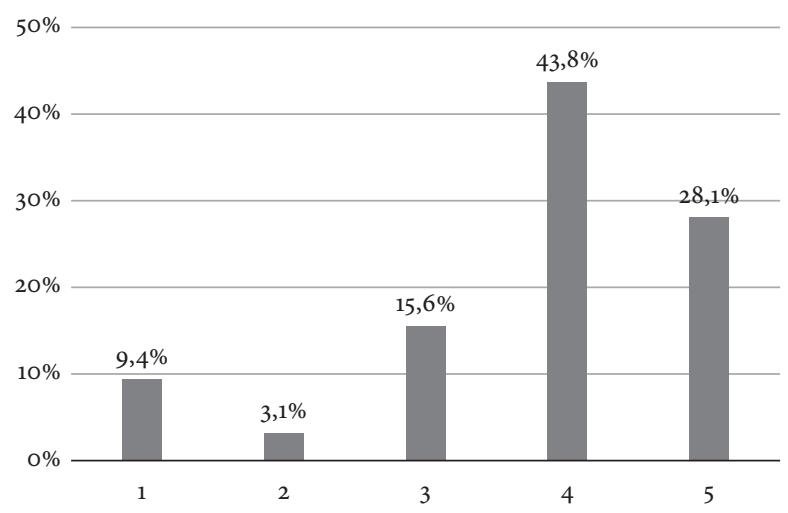

Figure 10. Most of the students found the exercise "useful" or "very useful." 
is strictly related to design education. The question "Did you learn something beyond information visualization creating a diagram for Wikipedia?" produced several insights. Many students said it was simpler than they thought it would be:

We never think about it, but it should be something to do more often, it is something for everybody. (Anonymous student \#10)

Other students were impressed in realizing how many people read Wikipedia, and therefore see their diagrams:

I learnt something new not related to design. It is nice to think that many people around the world will see my diagram. (Anonymous student \#12)

Only one student cited the community feedback as something enriching:

The positive comments left by other users on the utility of my diagram and its reuse on other language editions made me understand the relevance of graphic design and its power in transforming culture (Anonymous student \#32)

Being forced to expose their work to unknown people, and receiving in most cases positive reactions, made clear to our students their role as communication designers in the dissemination of knowledge.

Even if most of the students followed the fate of their diagram after the end of the course (75\%), and most found the experience interesting, when asked if they continued to contribute to Wikipedia, only one answered affirmatively.

\section{Discussion}

As we stated in the introduction, our aim was twofold: the definition of a new format for the education of communication designers, and the analysis of how information designers can act on Wikipedia. As a didactical activity, the experience proved to be valuable from several perspectives. Students felt that their work was useful for other people, and not just to improve their visual skills in information design. The feedback gathered from external people, not necessarily experts in graphic design, were useful to test their ability to convey meaningful information. The experience helped them to reflect on the role of designers in communicating information and knowledge as well as on the tradeoff among visual clarity and information richness. Finally, it was an opportunity for them to understand what a collaborative open project is while contributing to it.

Some issues remain unresolved. Since for the students it was an exercise, their approach was more focused on convincing other users to accept the visualization rather than to engage in a discussion, at the cost of refining and modifying the diagram. We also found it difficult to keep our students engaged in the discussion with other Wikipedia users. Our students are more familiar with a "finish first, then publish" process. Diagrams, and artifacts in general, are released only when complete and fine-tuned. Adopting an open source mentality, based on the disclosure of the production process (Raymond 1999), is key if we want to contribute to collaborative processes as graphic designers. The approaches to establishing this mentality in our school are yet to be defined. Planning a future edition of this didactic exercise, we would try to involve other users as tutors for our students, guiding them in the policies of the encyclopedia. We would also set up a process based on quick releases, focusing on the discussion with other users toward improvement of the diagrams. 
As an experiment meant to bring information designers to Wikipedia, the experience revealed several limits and, at the same time, opened interesting reflections. It was difficult to evaluate the high rate of acceptance and low level of discussion by other Wikipedia users, which led to four hypotheses. First, it could be a tacit approval of the produced diagrams. Second, it could be related to the selection of marginal articles by our students, leading to a low interest for other users. A third hypothesis is that the addition or modification of images is seen as a marginal activity, less relevant than textual edits. Finally, since images are seen as difficult to edit, the effort is rewarded to encourage further contributions. More research is required to evaluate these hypotheses and to analyze the level of acceptance of visual edits compared to textual ones.

Another critical point is that almost none of our students continued to edit Wikipedia. Nevertheless, all of them declared the experience to be interesting and enriching. In this case, it is also difficult to evaluate the outcome. It could be a matter of time (the creation of a diagram, its test on the Wikipedia platform, and the discussion are all time-consuming), or a lack of reward for the effort. In our experience, young graphic designers are really motivated to work on a personal project when this brings visibility, adding it to their portfolio. In a system that discourages the creation of diagrams and diminishes the relevance of its author, the commitment could be easily lost. Lastly, a motivation could be a lack of a community of users focused on the creation of diagrams and visualization: information designers could feel lost, with no clear guidance and without an overall view of the project.

From a technical point of view, the creation and editing of an image proved to be complex and intricate. As we stated in the introduction, the openness of a diagram is not just related to the license one adopts, but also to the technology. If we want to enable other people to edit and modify our work, we should provide files designed to be modified: for example, favoring the use of vector images over raster ones.

Through this experiment we also observed a lack of tools to keep diagrams open and simple to edit. The main limitation is the difficulty in sharing an editable version of the diagram: while it is possible to upload editable files, their rendering and use across Wikipedia is highly unstable. Many students, in the end, opted for the raster images to preserve the visual appearance of the diagram. The overall architecture, in practice, seems to discourage the creation of diagrams that are simple to edit and update. Even if the project ethic is to produce artifacts meant to be free, reusable, and remixable, there are no formalized tools to enable this approach. In this experiment, we repurposed tools provided by MediaWiki (i.e., page history, talk page, Commons pages) to see the evolution of the diagrams produced by our students, but there is a lack of dedicated tools for images. If the Wikipedia community wants to extend to images the same ethics applied to texts, there is a need for new tools to keep track of reuses and transformations of images. As an example, taking inspiration from the $\mathrm{Git}^{5}$ environment, it could be useful to have tools to "fork" existing media and reuse them in a new artifact, keeping track of the links.

In general, we felt that, from a technological point of view, the platform is still highly focused on texts to the detriment of other media. Moreover, the problem for videos and non-static artifacts remains: if we represent something through an animation, how can we give other people the ability to modify it? How can we provide access to the source files, particularly when they have been created with proprietary software?

Even from the point of view of Wikipedia's community policies, the discussions show how users struggled in trying to understand how to apply existing policies to images. While for some policies the interpretation is 
quite simple (e.g., "Be bold," meaning The Wikipedia community encourages users to be bold when updating the encyclopedia), others are not. For example, how does the "no original research" apply to images? How to distinguish visual representations that are direct mappings of existent information from those that are original research? Also related to sources, there is a need for reflection on how they could be cited in a diagram.

To conclude, while the experience proved to be interesting and valuable for design education, a great deal of effort is still required both to involve information designers and to enable the creation of truly open diagrams on Wikipedia. While a strong community is guiding the textual enrichment of the encyclopedia, there is not yet any form of organized support for its visual dimension.

In our opinion, there are two ways to address such challenges. The first one is technological, and it relates to Wikipedia/Wikimedia: it should provide tools for sharing source files, not just the end results. The second is conceptual and concerns information designers: they should move from the idea of being authors to the idea of being contributors.

\section{Submission date: 10 October, 2016 \\ Accepted date: 8 February, 2017}

\section{Notes}

1. It is possible to see the full list of requested diagrams visiting this page: https://en.wikipedia.org/wiki/Category:Wikipedia_ requested_diagram_images

2. At the moment of writing, the Wikimedia Foundation operates 12 projects; here is possible to see the full list: https:// en.wikipedia.org/wiki/Wikipedia:Wikimedia_sister_projects

3. According to Wikipedia policies, an edit war occurs when editors who disagree about the content of a page repeatedly override each other's contributions.
4. The results of the exercise can be found at this link: https:// commons.wikimedia.org/wiki/Category:Contents_produced_ by_DensityDesign_students

5. Version control is a system that records changes to a file or set of files over time so that it is possible recall specific versions later (Chacon \& Straub 2014).

\section{References}

Alexa Internet Inc. (2016). The top 500 sites on the web. Retrieved October 5, 2016, from http://www.alexa.com/topsites/global

Bao, P., Hecht, B., Carton, S., Quaderi, M., Horn, M. \& Gergle, D. (2012). Omnipedia: bridging the Wikipedia language gap. In Proceedings of the 2012 ACM annual conference on Human Factors in Computing Systems - CHI '12 (p. 1075). New York, New York, USA: ACM Press. doi: 10.1145/2207676.2208553

Borra, E., Laniado, D., Weltevrede, E., Mauri, M., Magni, G., Venturini, T., Ciuccarelli, P., Rogers, R. \& Kaltenbrunner, A. (2015). A platform for visually exploring the development of Wikipedia articles. Proceedings of the gth International AAAI Conference of Web and Social Media.

Chacon, S. \& Straub, B. (2014). Pro Git (2nd ed.). Berkeley, CA: Apress. doi: 10.1007/978-1-4842-0076-6

List of Wikipedias. (2016). Retrieved October 5, 2016, from https:// en.wikipedia.org/wiki/List_of_Wikipedias

Massa, P. \& Scrinzi, F. (2013). Manypedia: Comparing language points of view Wikipedia communities. First Monday, 18(1). doi: $10.1145 / 2462932.2462960$

Mauri, M. (2011). Share your knowledge: Mapping project's influences on the web. Retrieved September 30, 2016, from http://www.densitydesign.org/research/share-yourknowledge-mapping-project's-influences-on-the-web/

Raymond, E. (1999). The cathedral and the bazaar. Knowledge, Technology \{\&\} Policy, 12(3), 23-49. article. doi: 10.1007/s12130-999-1026-0

Ricci, D. (2010). Seeing what they are saying: Diagrams for sociotechnical controversies. In D. Durling, R. Bousbaci, L.-L. Chen, P. Gauthier, T. Poldma, S. Rowoth-Stokes \& E. Stolterman (Eds.), DRS2010-Design \& Complexity. Montréal.

Ricci, D., Ciuccarelli, P. \& Valsecchi, F. (2008). Handling changes through diagrams: Scale and grain in the visual 
representation of complex systems. In C. Cipolla \& P.P. Peruccio (Eds.). Turin: Allemandi.

State of the Commons. (2015). Retrieved October 5, 2016, from https://stateof.creativecommons.org/2015/

Viegas, F. (2007). The visual side of Wikipedia. In 2007 4oth Annual Hawaii International Conference on System Sciences (HICSS'07) (pp. 85-85). IEEE. doi: 10.1109/HICSS.2007.559

Wattenberg, M., Viégas, F.B. \& Hollenbach, K. (2007). Visualizing activity on Wikipedia with chromograms. Methods, 4663/2010, 272-287. doi: 10.1007/978-3-540-74800-7_23

WikiEdu Website. (n.d.). Retrieved October 5, 2016, from https:// wikiedu.org/

Wikipedia: No original research. (2016). Retrieved October 5, 2016, from https://en.wikipedia.org/wiki/Wikipedia:No_ original_research

Wikipedia: Policies and guidelines. (2016). Retrieved October 5 , 2016, from https://en.wikipedia.org/wiki/Wikipedia:Policies_ and_guidelines

\section{About the authors}

Michele Mauri is research fellow at

Politecnico di Milano. Collaborating with DensityDesign Research Lab since 2009, he obtained his $\mathrm{PhD}$ with the dissertation The design of un-finished. Key publication are related to the visual analysis of Wikipedia (Societal Controversies in Wikipedia Articles), on the role of designers in the communication of complex phenomena (Designing Controversies and Their Publics, Designing diagrams for social issues) and on visual interfaces for the exploration of cultural corpora (Weaving data, slicing views).

Email: michele.mauri@polimi.it
Azzurra Pini is a research fellow with the DensityDesign Research Lab (www.densitydesign.org) at Politecnico di Milano, Italy. Azzurra is a communication designer and her $\mathrm{PhD}$ research has been focusing on the role of visualization for the design of visual interfaces for exploring, analysing and designing organisational processes to assist researchers, analysts, and managers in their inquiry activity. Her current research interests pertain to all fields of application of communication and information design, including interdisciplinary research on complex phenomena and decision-support by means of visualization.

Paolo Ciuccarelli is an Associate Professor at Politecnico di Milano, he teaches at the Faculty of Design in the Communication Design master degree. He has been visiting lecturer at Universidad de Malaga and The Royal Institute of Art (KKH—Stockholm) and participated to several postgraduate courses in other universities. Head of the

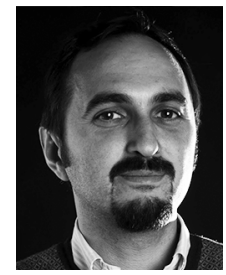
Communication Design Research group and member of the Design PhD board, both at Politecnico di Milano-Design Department. His research and publishing activities focus on the development of data, information and knowledge visualization tools and methods to support decision making processes in complex systems. 